Louisiana State University

LSU Digital Commons

4-7-2009

\title{
Calculation of water equivalent thickness of materials of arbitrary density, elemental composition and thickness in proton beam irradiation
}

\author{
Rui Zhang \\ University of Texas Graduate School of Biomedical Sciences at Houston \\ Wayne D. Newhauser \\ University of Texas Graduate School of Biomedical Sciences at Houston
}

Follow this and additional works at: https://digitalcommons.Isu.edu/physics_astronomy_pubs

\section{Recommended Citation}

Zhang, R., \& Newhauser, W. (2009). Calculation of water equivalent thickness of materials of arbitrary density, elemental composition and thickness in proton beam irradiation. Physics in Medicine and Biology, 54 (6), 1383-1395. https://doi.org/10.1088/0031-9155/54/6/001

This Article is brought to you for free and open access by the Department of Physics \& Astronomy at LSU Digital Commons. It has been accepted for inclusion in Faculty Publications by an authorized administrator of LSU Digital Commons. For more information, please contact ir@lsu.edu. 


\title{
Calculation of water equivalent thickness of materials of arbitrary density, elemental composition and thickness in proton beam irradiation
}

\author{
Rui Zhang ${ }^{1,2}$ and Wayne D Newhauser ${ }^{1,2,3}$ \\ Wayne D Newhauser: wnewhaus@mdanderson.org \\ ${ }^{1}$ Graduate School of Biomedical Sciences at Houston, The University of Texas, 6767 Bertner, \\ Houston, TX 77030, USA \\ ${ }^{2}$ Department of Radiation Physics, Unit 94, The University of Texas M. D. Anderson Cancer \\ Center, 1515 Holcombe Boulevard, Houston, TX 77030, USA
}

\section{Abstract}

\begin{abstract}
In proton therapy, the radiological thickness of a material is commonly expressed in terms of water equivalent thickness (WET) or water equivalent ratio (WER). However, the WET calculations required either iterative numerical methods or approximate methods of unknown accuracy. The objective of this study was to develop a simple deterministic formula to calculate WET values with an accuracy of $1 \mathrm{~mm}$ for materials commonly used in proton radiation therapy. Several alternative formulas were derived in which the energy loss was calculated based on the Bragg-Kleeman rule (BK), the Bethe-Bloch equation (BB) or an empirical version of the BetheBloch equation (EBB). Alternative approaches were developed for targets that were 'radiologically thin' or 'thick'. The accuracy of these methods was assessed by comparison to values from an iterative numerical method that utilized evaluated stopping power tables. In addition, we also tested the approximate formula given in the International Atomic Energy Agency's dosimetry code of practice (Technical Report Series No 398, 2000, IAEA, Vienna) and stopping power ratio approximation. The results of these comparisons revealed that most methods were accurate for cases involving thin or low- $Z$ targets. However, only the thick-target formulas provided accurate WET values for targets that were radiologically thick and contained high- $Z$ material.
\end{abstract}

\section{Introduction}

In many cases, proton radiation therapy provides lower integral dose to the patient compared to other external beam modalities. In particular, the finite range of a proton beam may be adjusted such that the therapeutic dose vanishes beyond the tumor. The proton range, which is commonly expressed in terms of its equivalent range in liquid water, may be precisely controlled by the accelerator (Coutrakon et al 1997), energy degrader system (Jongen et al

\footnotetext{
(C) 2009 Institute of Physics and Engineering in Medicine
}

${ }^{3}$ Author to whom any correspondence should be addressed. 
1996), a range shifter in the treatment head (Newhauser et al 2007b) and a range compensator (Wingate et al 1977, Fontenot et al 2007).

In proton therapy, water provides an excellent tissue-like phantom material for measurement of beam range and absorbed dose (International Commission on Radiation Units and Measurements (ICRU) 1998). The beam penetration range, as well as the thickness of components in the proton beam path, is commonly prescribed in terms of its penetration depth in liquid water, or water equivalent thickness (WET). In some situations, it is convenient to specify the water equivalent ratio (WER), which is the ratio of WET to material thickness, because WER values are approximately constant for some target thicknesses and beam energies and easier for comparison of results under different measurement or calculation conditions.

For clinical applications in proton therapy, WET values were traditionally measured by observing the range shift associated with the introduction of a test object, such as an immobilization device. In this technique, a pair of otherwise identical measurements yields the range shift in a water phantom, which is equal to the WET (Moyers 1999, Moyers et al 1992, Schneider et al 2002). In addition, several techniques have been suggested for calculating WET values. The International Atomic Energy Agency (IAEA) proposed an approximate technique to predict WET using the ratio of continuous-slowing-down approximation (CSDA) ranges (in $\mathrm{g} \mathrm{cm}^{-2}$ ) in water and the material of interest, and provided WET data for polymethylmethacrylate (PMMA) and polystyrene (IAEA 2000). Newhauser (2001) reported exact and approximate methods for calculating WET values along with WET data for PMMA and A-150 plastic. Newhauser et al (2007a) proposed a numerical method to calculate WET and WER values for gold, stainless steel and titanium. Their results revealed that WER calculations depend on proton energy and thickness, especially for high-density objects such as implanted fiducial markers and components in the treatment head (WER values decrease $1.1 \%$ from $0.1 \mathrm{~cm}$ to $0.5 \mathrm{~cm}$ stainless steel for 60 $\mathrm{MeV}$ proton beam, and increase $7.3 \%$ from $30 \mathrm{MeV}$ to $250 \mathrm{MeV}$ proton beam for $0.1 \mathrm{~cm}$ stainless steel). This is important in that many routine clinical tasks require high-accuracy WET calculations involving high-density materials. However, prior to the current work, these calculations required either iterative numerical methods or approximate methods of unknown accuracy.

The aim of this work was to develop and validate analytical expressions to calculate the WET of materials of arbitrary density, elemental composition and thickness for proton beam energies of up to $250 \mathrm{MeV}$. The expressions should be simple, convenient to use and accurate to within $1 \mathrm{~mm}$, i.e. small in comparison to the component of the distal margin associated with range uncertainties. The approach we used was to calculate the energy loss in the material of interest using expressions based on theoretical range-energy relations.

\section{Methods and materials}

Given a slab of material with thickness $t_{m}$ and mass density $\rho_{m}$, a proton beam with initial energy $E_{i}$ and a residual range $R$ in water, the WET of the material is the thickness of water 
$\left(t_{w}\right)$ that results in the same $R$. The unitless quantity WER is the ratio of $t_{w}$ to $t_{m}$. Figure 1 shows the WET concept schematically.

The general equation for WET is

$$
t_{w}=t_{m} \frac{\rho_{m}}{\rho_{w}} \frac{\bar{S}_{m}}{\bar{S}_{w}}
$$

where $\rho_{w}$ and $\rho_{m}$ are the mass densities of water and material, respectively, and $S_{m}^{-}$and $S_{\mathrm{w}}^{-}$ are the mean proton mass stopping power values for the material and water, respectively, defined by

$$
\bar{S}=\frac{\int_{E} S \mathrm{~d} E}{\int_{E} \mathrm{~d} E} .
$$

It is convenient to classify targets arbitrarily as being radiologically 'thick' or as being radiologically 'thin'. We shall define the special case of a thin target as a target in which the proton beam loses only a small fraction of its energy. In contrast, we define a thick target as a target in which the proton energy loss is sufficiently large. In principle, classifying a target as the radiologically 'thick' or 'thin' depends on the initial beam energy, the material composition, the material density and the physical thickness. For simplicity, we define the thin target exclusively in terms of fractional energy loss that is less than a constant, or

$$
\frac{\Delta E}{E_{i}}=\frac{E_{i}-E_{f}}{E_{i}}<\delta
$$

where $\delta$ is the energy-loss constraint with a typical value of 0.3 ( $\delta$ is discussed in detail in section 3). For 'thin' targets, a point value of $S\left(E_{i}\right)$ can be used in equation (1) instead of the mean value $S$.

In order to investigate the influence of the target material on the WET calculation, different targets of lead $\left(\rho=11.3 \mathrm{~g} \mathrm{~cm}^{-3}\right)$, aluminum $\left(\rho=2.70 \mathrm{~g} \mathrm{~cm}^{-3}\right)$ and PMMA $(\rho=1.19 \mathrm{~g}$ $\mathrm{cm}^{-3}$ ), respectively, representing high-, medium-, and low-density materials, were used in the theoretical calculations.

\subsection{Numerical method}

The numerical method of WET calculation (Newhauser et al 2007a), denoted in this work by NM, was used as the standard of comparison for both thin and thick targets for several reasons: first, NM can calculate many more data than measurement because it can deal with any thickness and any material, while it's difficult to measure all of them; second, NM is the most accurate calculation method so far, while single measurement may comprise the accuracy; third, the purpose of this paper is to simplify the math process to reproduce results, NM is an independent crosscheck. In this method, the path-length-averaged mass stopping power $(S)$ is defined as 


$$
\bar{S}=\frac{\int_{t} S \mathrm{~d} x}{\int_{t} \mathrm{~d} x} .
$$

The thickness of the water slab, $t_{w}$, was needed as a limit of integration. To solve this problem, we iteratively determined $t_{w}$ that yielded the same proton energy loss that occurred in targets by finding the root of

$$
f\left(\rho_{w} t_{w}\right)=\Delta E_{w}\left(\rho_{w} t_{w}\right)-\Delta E_{m}\left(\rho_{m} t_{w}\right)=0,
$$

where the energy loss in the slabs was calculated with

$$
\Delta E(\rho t)=\bar{S} \rho t .
$$

Despite its accuracy, this method is computationally intensive, and therefore, it is not very suitable for some clinical applications.

\subsection{Thin-target approach}

In the following sections, four methods are presented for calculating WET values for radiologically 'thin' targets. These methods do not consider the energy change of proton beam in the targets.

2.2.1. Bragg-Kleeman rule-The relationship between energy and range of proton beams in any given material can be determined using the Bragg-Kleeman (BK) rule (Bragg and Kleeman 1905):

$$
R=\alpha E^{p}, \quad \text { (7) }
$$

where $a$ is a material-dependent constant, $E$ is the initial energy of the proton and $p$ is an energy-dependent parameter. A special case of equation (7) is Geiger's rule with $p=1.5$, which is valid for protons that have an energy up to about $10 \mathrm{MeV}$ (Evans 1982). For simplicity, we used $p$ as a material-dependent constant in this study. The accuracy of equation (7) has been discussed by several studies (Bortfeld 1997, Ulmer 2007).

From equation (7), we can derive the expression for the point value mass stopping power,

$$
S=-\frac{\mathrm{d} E}{\rho \mathrm{d} x} \approx \frac{E^{1-p}}{\rho \alpha p}
$$

with $a$ and $p$ values obtained by fitting to either residual ranges or stopping powers data (Ziegler et al 1985). Substituting equation (8) into equation (1), we obtain the thin-target approximation, or 


$$
t_{w} \approx t_{m} \frac{\rho_{m}}{\rho_{w}} \frac{S_{m}}{S_{w}}=t_{m} \frac{\alpha_{w} p_{w}}{\alpha_{m} p_{m}} E^{p_{w}-p_{m}}
$$

with initial energy $E_{i}$ used for the $E$ value.

2.2.2. Bethe-Bloch and empirical Bethe-Bloch equations-The Bethe-Bloch (BB) equation (Bethe 1930, Bloch 1933) for the energy loss of heavy charged particles is

$$
-\frac{\mathrm{d} E}{\rho \mathrm{d} x}=4 \pi N_{A} r_{e}^{2} m_{e} c^{2} z^{2} \frac{Z}{A} \frac{1}{\beta^{2}}\left[\frac{1}{2} \operatorname{In} \frac{2 m_{e} c^{2} \gamma^{2} \beta^{2} T_{\max }}{I^{2}}-\beta^{2}-\frac{\delta(\beta)}{2}-\frac{C(\beta)}{Z}\right],
$$

where $N_{A}$ is Avogadro's number, $r_{e}$ is the classical electron radius, $m_{e}$ is the mass of electron, $Z$ is the atomic number of absorbing material, $A$ is the atomic weight of absorbing material, $z$ is the charge of incident particle, $\beta=v / c$ where $v$ is the velocity of the incident particle, $\gamma=\left(1-\beta^{2}\right)^{-1 / 2}$, and $T_{\max }$ is the maximum energy transferred to a single electron and is defined by

$$
T_{\max }=2 m_{e} c^{2} \beta^{2} \gamma^{2}\left(1+2 \gamma \frac{m_{e}}{M}+\frac{m_{e}^{2}}{M^{2}}\right)^{-1}
$$

At low energy, $T_{\max } \approx 2 m_{e} c^{2} \beta^{2} \gamma^{2}$. Other variables in equation (10) are $I$, the mean excitation energy of the absorbing material; $\delta(\beta)$, the 'density correction' arising from the shielding of remote electrons by close electrons, which results in a reduction of energy loss for higher energies, and $C(\beta)$, the 'shell correction', which is only important for low energies where the particle velocity is of the same order of magnitude as the velocity of the atomic electrons. Because $\delta(\beta)$ and $\mathrm{C}(\beta)$ were approximately zero for all proton energies used in this study (Leo 1987), we ignored the last two items in the brackets of equation (10) for subsequent calculations.

For compounds, we need to know the mass fraction of each constituent element before we can calculate the effective mean excitation energy, $I_{\mathrm{eff}}$, for the mixture as

$$
\operatorname{In}\left(I_{\text {eff }}\right)=\sum \frac{a_{i} Z_{i} \operatorname{In}\left(I_{i}\right)}{\sum a_{i} Z_{i}}
$$

where $a_{i}, Z_{i}$, and $I_{i}$ are the number of atoms, the atomic number and the mean excitation energy of the $i$ th element in the compound, respectively (Leo 1987).

For thin targets, we derived the following formula for WET based on the BB equation:

$$
t_{w} \approx t_{m} \frac{\rho_{m}}{\rho_{w}} \cdot \frac{S_{m}}{S_{w}}=\left.t_{m}\left(\rho \frac{Z}{A} \cdot\left[\operatorname{In} \frac{2 m_{e} c^{2} \gamma^{2} \beta^{2}}{I}-\beta^{2}\right]\right)\right|_{w} ^{m} .
$$


The only uncertain item is $I$, which can be found in previously published work (Sternheimer et al 1984).

Instead of using tabular values for $I$, we proposed the following empirical form of the BB equation (EBB), in which $I$ for a single element is empirically calculated by $I=k Z$, and

$$
k=\left\{\begin{array}{ccc}
14.5, & \text { for } & Z \leq 8 \\
13, & \text { for } & 8<Z \leq 13 \\
11, & \text { for } & Z>13
\end{array}\right.
$$

This approach is similar to other previously reported empirical approaches (Bloch 1933, Sternheimer 1952, Barkas and Berger 1964, Mukherji 1975, Dehmer et al 1975) but has the advantage of both simplicity and, in principle, applicability to elements commonly found in proton therapy beams. In equation (14), it was assumed that $I$ depends weakly on the $Z$ value of the absorber element. The rationale for this approach was primarily to determine if the accuracy of the BB method could be improved upon by using an empirical and Z-dependent adjustment to the $I$ values.

2.2.3. Stopping power ratio (SPR) approximation-For thin targets, if the stopping powers for different point value energies are known, one can use the thin approximation of equation (1) to calculate WET directly, i.e.

$$
t_{w}=t_{m} \frac{\rho_{m}}{\rho_{w}} \frac{S_{m}\left(E_{i}\right)}{S_{w}\left(E_{i}\right)}
$$

where $E_{i}$ is the initial energy of a proton beam. For some energies, if stopping power data are not available, one can interpolate the stopping power data based on equation (8) by linearly fitting $\ln (S)$ over $\ln (E)$.

\subsection{Thick-target approach}

For arbitrarily thick targets, the energy changes cannot be ignored and the mean mass stopping powers in equation (1) must be calculated. Consequently, the equations used for the thin-target approach must be generalized to take into account the energy gradient through thick targets.

Accordingly, for the BK rule,

$$
\bar{S}=\frac{\int_{E_{i}}^{E_{f}}\left(E^{1-p}(\rho \alpha p)^{-1}\right) \mathrm{d} E}{\int_{E_{i}}^{E_{f}} \mathrm{~d} E}
$$

where $E_{i}\left(E_{f}\right)$ is the initial (final) energy. According to the BK rule, 


$$
E(R-x)=\left(\frac{R-x}{\alpha}\right)^{\frac{1}{p}}
$$

where $x$ is the distance that the incident particle travels in the material. So the final energy of the proton beam after penetrating the target is

$$
E_{f}=E\left(R-t_{m}\right)=\left(\frac{R-t_{m}}{\alpha}\right)^{\frac{1}{p}}
$$

By plugging equation (16) into equation (1), the expression of WET is

$$
t_{w}=t_{m}\left[\frac{\alpha_{w} p_{w}\left(2-p_{w}\right)}{\alpha_{m} p_{m}\left(2-p_{m}\right)}\right]\left(\frac{E_{f}^{2-p_{m}}-E_{i}^{2-p_{m}}}{E_{f}^{2-p_{w}}-E_{i}^{2-p_{w}}}\right) .
$$

For the BB equation, the mean mass stopping power can be approximated as

$$
\bar{S}=\frac{4 \pi N_{A} r_{e}^{2} m_{e} c^{2} z^{2} \int_{E_{i}}^{E_{f}}\left(\frac{z}{A \beta^{2}}\left[\operatorname{In}\left(\frac{2 m_{e} c^{2} \gamma^{2} \beta^{2}}{I}\right)-\beta^{2}\right]\right) \mathrm{d} E}{\int_{E_{i}}^{E_{f}} \mathrm{~d} E}
$$

where $E_{i}$ is the initial energy; the final energy can be calculated by $E_{f}=E\left(R-t_{m}\right)$. After getting the numerical values of mean mass stopping powers for the target and water, WET can be calculated by plugging equation (20) into equation (1).

The IAEA (2000) proposed that WET can be approximated by

$$
t_{w}=t_{m} \frac{\rho_{m}}{\rho_{w}} c_{m}
$$

where the depth scaling factor, $c_{m}$, can be calculated, to a good approximation, as the ratio of the CSDA range (in $\mathrm{g} \mathrm{cm}^{-2}$ ) in water to that in the target:

$$
c_{m}=\frac{\rho_{w} R_{w}}{\rho_{m} R_{m}}
$$

Thus, equation (21) can be rewritten as

$$
t_{w} \approx t_{m} \frac{R_{w}}{R_{m}} .
$$

Because the range in equation (23) corresponds to the complete loss of ion energy, this approach may be considered a stopping-length-target approximation, which means that the target thickness must be larger than the beam range, so the application field of IAEA 
approximation is very narrow, while any target thickness can be calculated by using other thick-target approach.

Because there is no theoretical range-energy relation used to calculate energy loss in SPR approximations, it was not developed in the thick-target approach.

\section{Results}

We used the approach based on the thin-target approach to calculate the WET values of several targets, including $1.5 \mathrm{~cm}$ of lead, $1.5 \mathrm{~cm}$ of aluminum and $10 \mathrm{~cm}$ of PMMA. The corresponding best estimates of the true WET values for these slabs were calculated using the iterative numerical method (NM). The parameters used in the BK rule (see equation (7)) for different materials are listed in table 1. Figure 2 shows the ratios between the WET values calculated using the various methods to those calculated by the NM as a function of proton energy.

In this work, the analytical formulas to calculate WET were classified according to approximations in calculating the energy loss in the target; we also classified targets as radiologically 'thin' or 'thick' using a value of $\delta$ of 0.3 in equation (3). The thin-target approach generated much better results (which means to be closer to the results from standard NM) for radiologically 'thin' targets, in which the energy loss $\Delta E / E$ was small (open symbols in figure 2) compared to that of 'thick' targets (solid symbols in figure 2), especially for high density material (lead). The formulas based on the BK rule, the EBB equation, and SPR approximation generated better results than the approaches based on the BB equation.

The thick-target approach, based on the BK rule, BB equation and EBB equation, provided good results for all cases considered. Figure 3 shows the ratios between WET values calculated by different thick approach and those calculated by the NM as a function of proton energy. Compared to the thin approach (see figure 2), the thick approach gave overall better results for various materials at different energies, especially for 'thick' targets, except for IAEA approximation. For both radiologically 'thin' and 'thick' targets, the EBB equation using equation (14) generated the most accurate results for lead (figure 3(c)); the BK rule gave the most accurate results for low- and medium-density materials (figure 3(a)). Both of them gave better results than the BB equation (figure 3(b)). However, the performance of IAEA approximation was much worse than the other methods, especially for high $\mathrm{Z}$ material (figure 3(d)).

To compare the overall performance of the various methods, we plotted the maximum WET deviations, $\Delta R_{\max }$, where $\Delta R=\left|\mathrm{WET}-\mathrm{WET}_{\text {num }}\right|$, relative to the corresponding prediction from the NM for all proton energies used. $\Delta R$ may be considered as the 'range' error associated with the methods in the WET calculation. Figure 4 includes $\Delta R$ values from various calculation methods, target thicknesses, and target materials. For lead targets (figure 4(a)), the range errors associated with methods in the WET calculations were largetypically 1-3 $\mathrm{mm}$ for thin approach, less than $1 \mathrm{~mm}$ for thick approach. In PMMA and aluminum, the range errors were small-less than $1 \mathrm{~mm}$ in all cases considered. 
The largest deviations in WET calculation for any material at any given thickness almost always occurred when the beam was at the lowest possible initial energy. This is because the energy loss ratio is the largest near the threshold energy. The data showed that for mediumand low-density materials, the deviations in WET were always less than $1 \mathrm{~mm}$ (figure 4(b), (c)). Figure 5 shows the $\Delta R$ values using the thick-target approach for high-density material lead. If we increased the energy constraint to $\delta=0.7$, i.e., after raising the lowest initial energy, the $1 \mathrm{~mm}$ goal for the accuracy of estimates was achieved in all cases for the BK rule and $\mathrm{EBB}$ equation (but not for the BB equation).

Figure 6 and table 2 show the WER values calculated using the NM for different materials with different thicknesses. Those data reveal that WER values depend strongly on the target material, the beam energy and the target thickness. The WER values were higher for material with higher density for beams of the same energy. For the high-density material (lead), WER ranges from 5.409 for $110 \mathrm{MeV}$ proton energy to 5.748 for $250 \mathrm{MeV}$ proton energy, revealing its obvious dependence on the energy. WER also decreases slightly as the target thickness increased from $0.1 \mathrm{~cm}$ to $1.5 \mathrm{~cm}$ for the high-density material. For the medium-density material (aluminum), WER has a slight dependence on energy and target thickness at energies less than $100 \mathrm{MeV}$ but was almost independent of these factors at higher beam energies. For the low-density material (PMMA), WER is almost independent of proton energy and target thickness.

\section{Discussion}

We evaluated several methods to calculate WET and WER values for both radiologically 'thin' and 'thick' targets. For high-density material, we found that our EBB equation gave the most accurate results for WET values; for medium- and low-density materials, the BK rule performed better. For radiologically 'thin' targets, all the methods provided reasonable accuracy, except for IAEA approximation when applied to lead targets. For radiologically 'thick' targets, the thick-target approach generally provided better accuracy than the simpler thin-target approach.

Our findings suggest that selecting an appropriate approach to calculate WET values may be critical in some cases. We present the following examples as clinically relevant scenarios. If the clinical requirement on range accuracy is $1.5 \mathrm{~mm}$, then the range error associated with approximations in the WET calculation must be less than $1.5 \mathrm{~mm}$. Thus, the required accuracy of the WET value for a typical lead scattering foil could be met with the thicktarget approach based on the BK rule and the EBB equation while the other analytical approximations would introduce unacceptably large range errors. In contrast, if the accuracy requirement on WET for a $2 \mathrm{~cm}$ thick PMMA range compensator is $0.2 \mathrm{~mm}$, any of the approaches considered would yield satisfactory results.

The clinical significance of this work was that we showed that several simple methods could be used to calculate WET values with small, known errors. The calculation of WET is important for the configuration of treatment planning systems and for routine quality assurance procedures in medical physics. The analytical formulas developed in this work will facilitate the convenient and accurate calculation of WET values. 
The results of our study are consistent with previous investigations. For example, the dependence of WER values on proton energy and thickness, especially for high density objects, was similar to previous work (Newhauser et al 2007a). Our WER values for PMMA were consistent with that from the IAEA report (IAEA 2000) (1.160) and Newhauser (2001) (1.162). The relative linear stopping powers reported by Moyers (1999) for PMMA and aluminum were 1.162 and 2.114, respectively, for $200 \mathrm{MeV}$ proton beam, which are in good agreement with our WER values. The WER values for PMMA and aluminum reported by Schneider et al (2002) were 1.14 and 2.08 for $177 \mathrm{MeV}$ proton beam, which are also consistent with our results.

Our study has some limitations. First, we considered a limited number of target materials and thickness. Although the empirical method to calculate mean excitation energy worked reasonably well for the materials we considered, it is not known if this method is applicable to other materials (EBB is also applicable to gold and titanium, data not shown). Second, we looked at energy loss and range shift only; we intentionally excluded the dosimetric implications of multiple coulomb scattering and nuclear reactions. These may be important for proton beams of small lateral size (Schneider et al 2002). Third, as the results showed, calculations from the BB equation had much larger errors than the other methods, which may due to the uncertainty in the mean excitation energy values.

\section{Acknowledgments}

We would like to thank Phillip Taddei for helpful discussions; Kathryn Carnes for assistance in preparing the manuscript. This work was supported in part by Northern Illinois University through a subcontract of Department of Defense contract W81XWH-08-1-0205.

\section{References}

Barkas, WH.; Berger, MJ. Tables of energy losses and ranges of heavy charged particles NASA SP-3013. Scientific and Technical Information Division, National Aeronautics and Space Administration; Washington, DC: 1964.

Bethe H. Zur Theorie des Durchgangs schneller Korpuskularstrahlen durch Materie. Ann Phys. 1930; 5:324-400.

Bloch F. Zur Bremsung rasch bewegter Teilchen beim Durchgang durch Materie. Ann Phys. 1933; 16:285-320.

Bortfeld T. An analytical approximation of the Bragg curve for the therapeutic proton beams. Med Phys. 1997; 24:2024-33. [PubMed: 9434986]

Bragg W, Kleeman R. On the alpha particles of radium and their loss of range in passing through various atoms and molecules. Phil Mag. 1905; 10:318-40.

Coutrakon G, Cortese, Ghebremedhin A, Hubbard J, Johanning J, Koss P, Maudsley G, Slater CR, Zuccarelli C, Robertson J. Microdosimetry spectra of the Loma Linda proton beam and relative biological effectiveness comparisons. Med Phys. 1997; 24:1499-506. [PubMed: 9304579]

Dehmer JL, Inokuti M, Saxon RP. Systematics of moments of dipole oscillator-strength distributions for atoms of the first and second row. Phys Rev A. 1975; 12:102-21.

Evans, RD. The Atomic Nucleus. Malabar, FL: Krieger; 1982.

Fontenot JD, Newhauser WD, Bloch C, White RA, Titt U, Starkschall G. Determination of output factors for small proton therapy fields. Med Phys. 2007; 34:489-98. [PubMed: 17388166]

IAEA. IAEA Technical Report Series 398. Vienna: International Atomic Energy Agency; 2000. Absorbed Dose Determination in External Beam Radiotherapy. 
ICRU. ICRU. Bethesda, MD: ICRU; 1998. Clinical Proton Dosimetry: Part I. Beam Production, Beam Delivery and Measurement of Absorbed Dose. Report 59

Jongen Y, Beeckman W, Cohilis P. The proton therapy system for MGH's NPTC: equipment description and progress report. Bull Cancer Radiother. 1996; 83(Suppl):219s-22s. [PubMed: 8949784]

Leo, WR. Techniques for Nuclear and Particle Physics Experiments. Berlin: Springer; 1987.

Moyers, MF. The Modern Technology of Radiation Oncology: A Compendium for Medical Physicists and Radiation Oncologists. Dyk, JV., editor. Wisconsin: Medical Physics Publishing; 1999. chapter 20

Moyers MF, Miller DW, Siebers JV, Galindo R, Sun S, Sardesai M, Chan L. Water equivalence of various materials for 155 to $250 \mathrm{MeV}$ protons. Med Phys. 1992; 19:829.

Mukherji S. Calculation of the mean ionization potentials of the elements for stopping-power computations. Phys Rev B. 1975; 12:3530-2.

Newhauser, W. Massachusetts General Hospital Report HD-112. Boston: 2001. Dosimetry for the gantry beams at the northeast proton therapy center: Part I Dimensions and geometric relationships.

Newhauser W, Fontenot J, Koch N, Dong L, Lee A, Zheng Y, Waters L, Mohan R. Monte Carlo simulations of the dosimetric impact of radiopaque fiducial markers for proton radiotherapy of the prostate. Phys Med Biol. 2007a; 52:2937-52. [PubMed: 17505081]

Newhauser W, Fontenot J, Zheng Y, Polf J, Titt U, Koch N, Zhang X, Mohan R. Monte Carlo simulations for configuring and testing an analytical proton dose-calculation algorithm. Phys Med Biol. 2007b; 52:4569-84. [PubMed: 17634651]

Schneider U, Pemler P, Besserer J, Dellert M, Moosburger M, de Boer J, Pedroni E, Boehringer T. The water equivalence of solid materials used for dosimetry with small proton beams. Med Phys. 2002; 29:2946-51. [PubMed: 12512731]

Sternheimer RM. The density effect for the ionization loss in various materials. Phys Rev. 1952; 88:851-9.

Sternheimer RM, Berger MJ, Seltzer SM. Density effect for the ionization loss of charged particles in various substances. At Data Nucl Data Tables. 1984; 30:261-71.

Ulmer W. Theoretical aspects of energy-range relations, stopping power and energy straggling of protons. Rad Phys Chem. 2007; 76:1089-1107.

Wingate CL, Archambeau JO, Koehler AM, Bennett GW. Proton penetration and control in nonhomogeneous phantoms. Med Phys. 1977; 4:198-201. [PubMed: 407434]

Ziegler, JF.; Biersack, JP.; Littmark, U. The Stopping and Range of Ions in Solids. New York: Pergamon; 1985. 


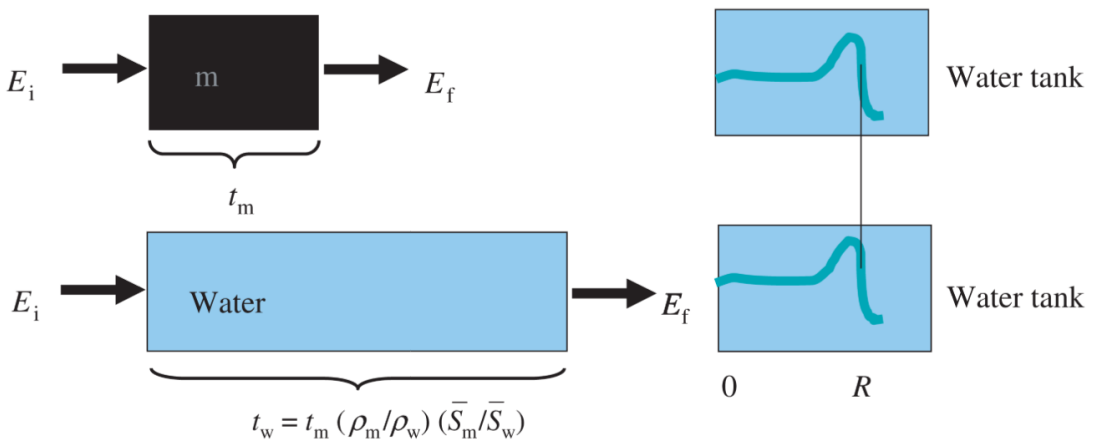

Figure 1.

Schematic illustration of the concept of WET. 


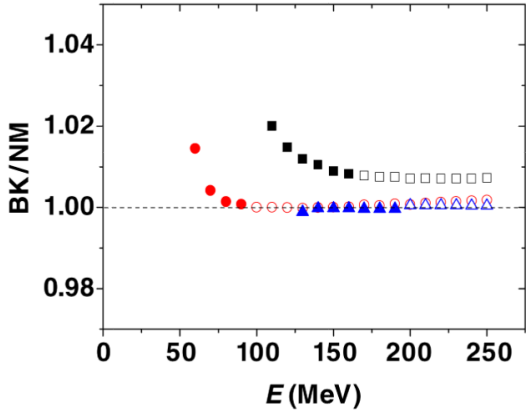

(a)

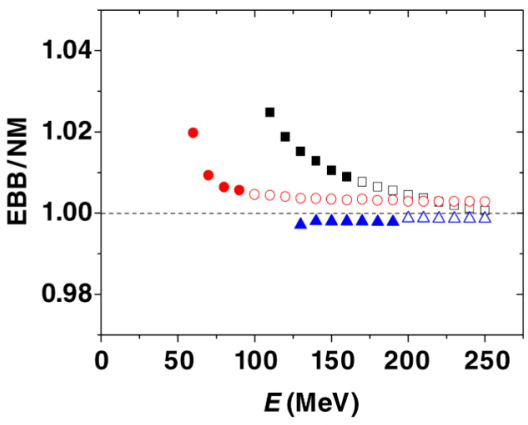

(c)

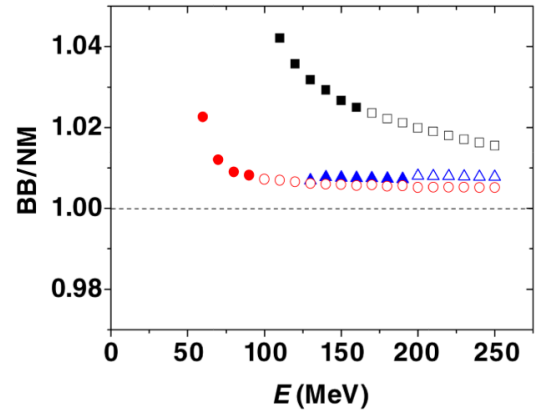

(b)

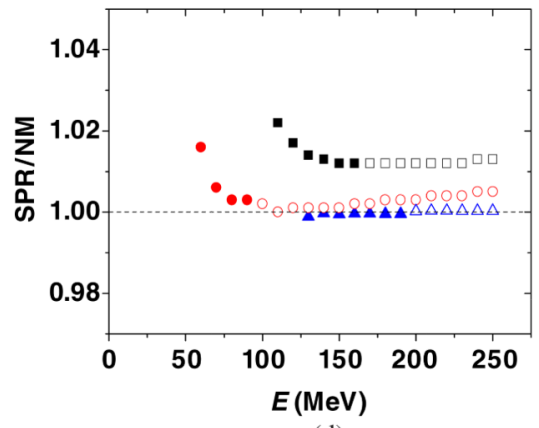

(d)

Figure 2.

The ratio of WET values calculated from thin approach (a) BK rule, (b) BB equation, (c) EBB equation and (d) SPR approximation to those of the NM as the function of energy for $1.5 \mathrm{~cm}$ lead (squares), $1.5 \mathrm{~cm}$ aluminum (circles) and $10 \mathrm{~cm}$ PMMA (triangles). Solid symbols stand for radiologically 'thick' targets while open symbols stand for radiologically 'thin' targets. 


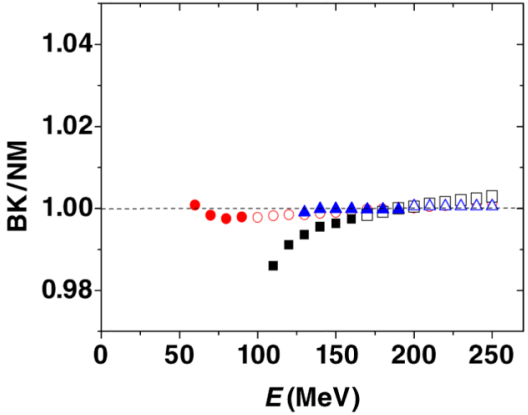

(a)

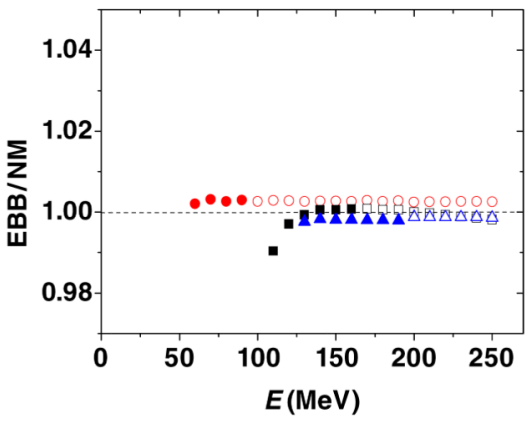

(c)

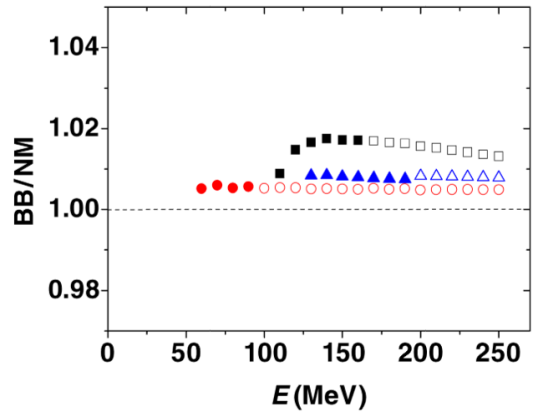

(b)

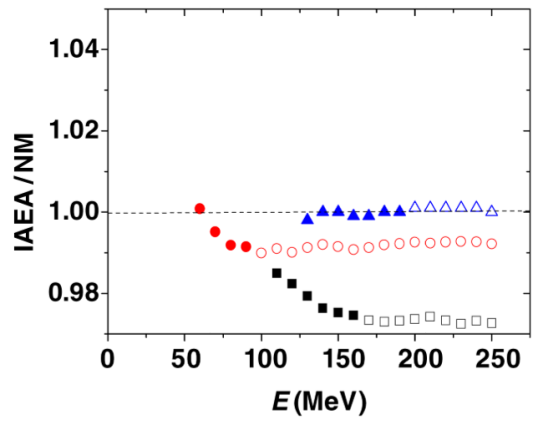

(d)

Figure 3.

The ratio of WET values calculated using the thick approach (a) BK rule, (b) BB equation, (c) EBB equation and (d) IAEA approximation to the NM as the function of energy for 1.5 $\mathrm{cm}$ lead (squares), $1.5 \mathrm{~cm}$ aluminum (circles) and $10 \mathrm{~cm}$ PMMA (triangles). Solid symbols stand for radiologically 'thick' targets while open symbols stand for radiologically 'thin' targets. 


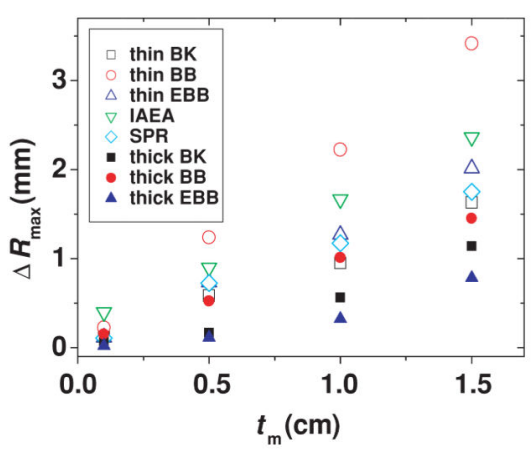

(a)

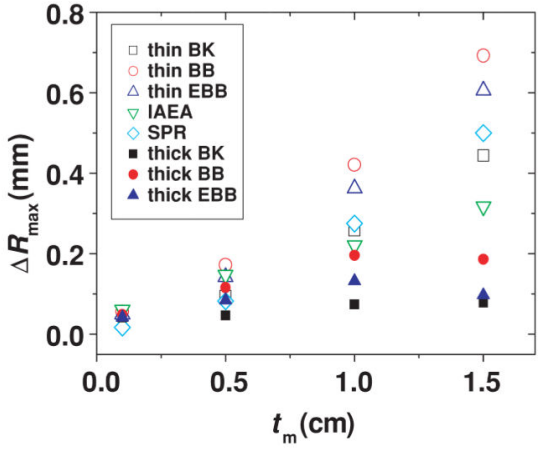

(b)

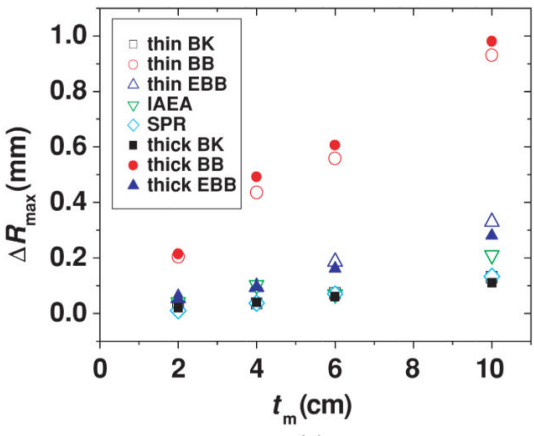

(c)

Figure 4.

Maximum WET deviation, $\Delta R_{\max }$, of different methods from the standard NM for (a) lead, (b) aluminum and (c) PMMA with different target thicknesses. 


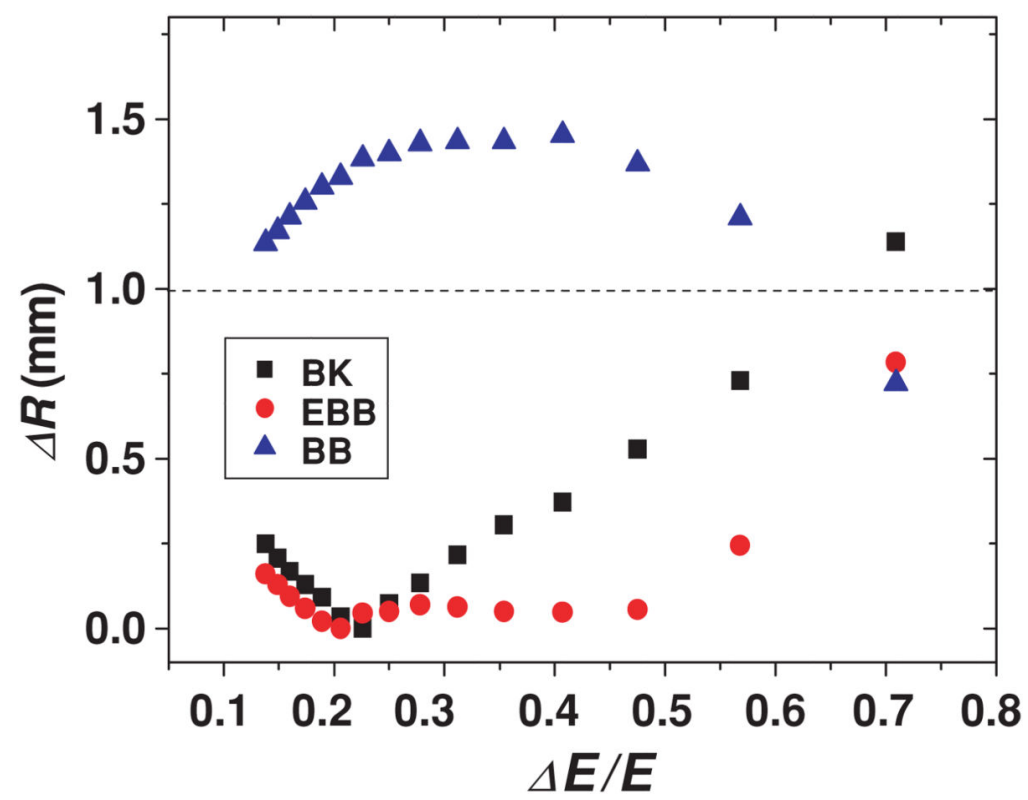

Figure 5.

WET deviation, $\Delta R$, using the thick-target approach for $1.5 \mathrm{~cm}$ lead as a function of energy loss. 


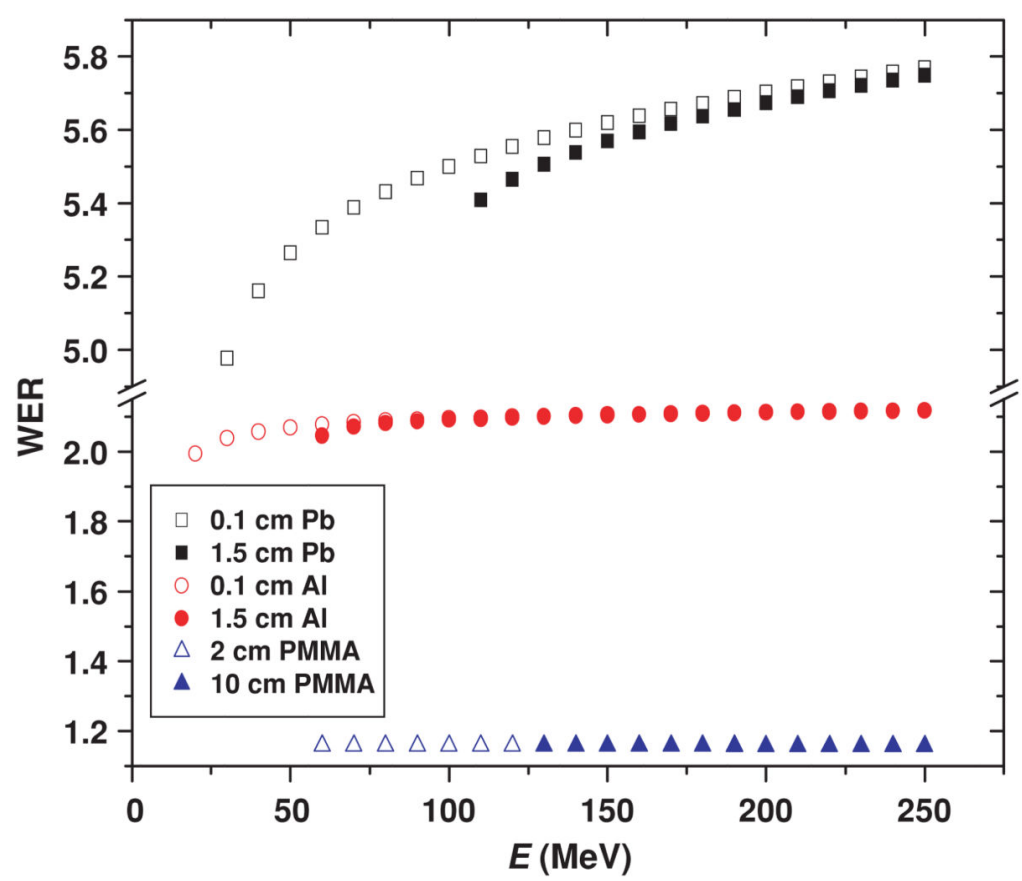

Figure 6.

Calculated water-equivalent ratio (WER) values by the NM as a function of proton beam energy. This plot illustrates the dependence of WER value on the target material, the beam energy and the target thickness. 
Table 1

Fitting parameters $a$ and $p$ for different materials for the BK rule. The energies for the proton beams used in the fit were from 10 to $250 \mathrm{MeV}$ and the range and energy data were taken from Ziegler et al (1985)

\begin{tabular}{lllll}
\hline & Lead & Aluminum & PMMA & Water \\
\hline$a$ & $6.505 \times 10^{-4}$ & $1.364 \times 10^{-3}$ & $2.271 \times 10^{-3}$ & $2.633 \times 10^{-3}$ \\
$p$ & 1.676 & 1.719 & 1.735 & 1.735 \\
\hline
\end{tabular}




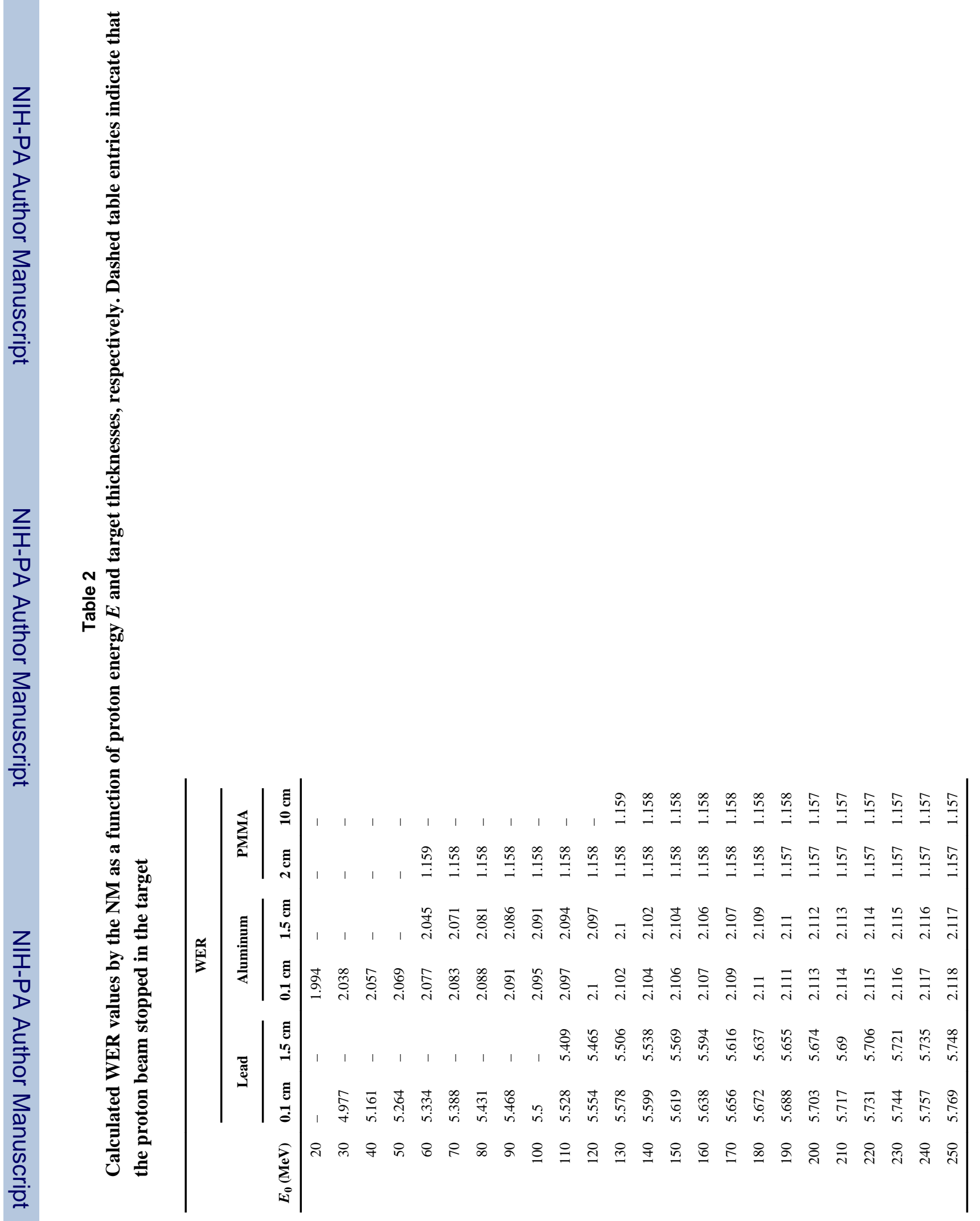

